June 1991

\title{
Psychiatry and Cardiac Transplantation
}

Mark Graham, M.D.

Case Western Reserve University, Cleveland, Ohio

Follow this and additional works at: https://jdc.jefferson.edu/jeffjpsychiatry

Part of the Psychiatry Commons

Let us know how access to this document benefits you

\section{Recommended Citation}

Graham, M.D., Mark (1991) "Psychiatry and Cardiac Transplantation," Jefferson Journal of Psychiatry. Vol. 9 : Iss. 2 , Article 4.

DOI: https://doi.org/10.29046/JJP.009.2.001

Available at: https://jdc.jefferson.edu/jeffjpsychiatry/vol9/iss2/4

This Article is brought to you for free and open access by the Jefferson Digital Commons. The Jefferson Digital Commons is a service of Thomas Jefferson University's Center for Teaching and Learning (CTL). The Commons is a showcase for Jefferson books and journals, peer-reviewed scholarly publications, unique historical collections from the University archives, and teaching tools. The Jefferson Digital Commons allows researchers and interested readers anywhere in the world to learn about and keep up to date with Jefferson scholarship. This article has been accepted for inclusion in Jefferson Journal of Psychiatry by an authorized administrator of the Jefferson Digital Commons. For more information, please contact: JeffersonDigitalCommons@jefferson.edu. 


\title{
Psychiatry and Cardiac Transplantation
}

\author{
Mark Graham, M.D.
}

\begin{abstract}
This paper explores the role of psychiatry in the field of cardiac transplantation by way of a literature review. The various stages of cardiac transplantation are discussed with respect to their time frame within the transplantation process, the cognitive and emotional reactions of patients within a given stage, and the dynamics of staff, patient, and family interactions. Research and clinical experience in the field suggest psychiatric assessment, follow-up, and, when necessary, intervention are valuable and important in promoting successful psychological adjustment as patients face their adaptive tasks in the various stages of the transplantation process.
\end{abstract}

\section{INTRODUCTION}

Since the first successful performance of cardiac transplantation in 1968 in South Africa, cardiac transplantation has steadily gained momentum. Improved medical technology has boosted postoperative survival rates and made transplantation a plausible option for those patients who are faced with endstage cardiac failure (1). The value and importance of routine psychiatric involvement in preoperative evaluation and screening as well as postoperative follow-up have been recognized since the beginning days of cardiac transplantation $(2,3)$.

One of the first papers written on the subject of psychiatric complications in cardiac transplant patients was by Dr. Donald Lunde in 1969 who at the time was a resident at Stanford University. In his article titled "Psychiatric Complications of Heart Transplants," Dr. Lunde notes a bizarre sidelight to the transplants that came as a surprise (4). After Stanford initiated its cardiac transplantation program, staff started receiving phone calls, letters, and visits to the emergency room at Stanford from people wanting to donate their hearts! These were usually people who were either profoundly depressed or seriously suicidal. Of course, those generous heart donations were declined and all the would-be donors were referred for psychiatric treatment. Since then we have learned a great deal more about the psychiatric aspects of cardiac transplantation (1).

The transplant process can be thought of as consisting of seven stages: transplant proposal, evaluation, waiting period, perioperative period, in hospital convalescence, discharge, and postdischarge adaptation (5). These stages represent milestones in the transplantation process and pose unique adaptive tasks for patients (5). Cardiac transplantation, like most types of major surgery, challenges the patient's ability to cope with preoperative stressors as well as those faced during postoperative recovery and rehabilitation. 
PRE-TRANSPLANT PERIOD

\section{Transplant Proposal}

During this stage, the patient is introduced to the idea of cardiac transplantation as a possible option for the treatment of his/her endstage cardiac disease. Patients are often taken by surprise when this idea is initially proposed and need time to think about it in order to make a decision (5). Patients whose cardiac problems have developed and progressed in a relatively short period of time may not have had a chance to adjust to their illness and may react to the transplant proposal with anger, disbelief, and refusal $(5,6)$. Those with a chronic illness course are usually excited at the chance to potentially beat their disease (5).

\section{Evaluation}

The stage of evaluation marks the beginning of a busy period in which there are two critical decisions to be made: patients need to give or withhold consent for transplant surgery and the transplant team must determine the eligibility of the patient (5,3). A full team evaluation may consist of medical, social, personal, compliance, and substance abuse history, medical examination, laboratory testing, psychiatric interview and mental status examination, and a variety of medical and psychological tests such as general health questionnaire, symptom checklist 90, MMPI, DIS, Milton behavioral health inventory (6,7,8,9). A typical multidisciplinary approach may include surgeons, internists, psychiatrists, psychologists, social workers and clinical nurse specialists $(7,10)$. Determination of patients' eligibility is made through consideration of patient's current cardiac status and the treatment options, overall medical condition, history of compliance with prescribed medical treatment, availability of family and social supports, and history of psychiatric problems including substance abuse $(7,9,10,11)$. Merrikin and Overcast indicate that the discretionary nature of the selection process may be discriminating against certain patients because doctors like to see good outcomes and may select patients who are least likely to suffer complications and have the best chance of adapting to the postoperative routine both physically and mentally (11).

Some programs have established absolute and relative contraindications to cardiac transplantation which include history of substance abuse or currently active substance abuse, history of noncompliance, history of clinical depression, borderline intellectual functioning or mental retardation, antisocial personality disorder, irreversible organic brain disease, and active psychosis $(2,7,8)$. Mai lists personality disorders, lesser degrees of substance dependence, and social isolation as relative contraindications which are not decisive alone but tend to discourage surgery in combination with each other (8). The reader will note that most, if not all, of the above-mentioned conditions can have a strong adverse effect on the patients' ability to comply with the prescribed medical treatment. Cooper, et al., followed 39 transplant patients postoperatively between 1974 and 1981; 9 of those 39 patients, had major complications secondary to noncompliance (12). Noncompliance appeared 
to be more common in single, divorced, and less educated patients. Cooper et al., state postransplantation challenges, unemployment, iatrogenic impotence, and family discord can lead to depression and noncompliance. They found patients with a high frustration tolerance and those with obsessive-compulsive traits tend to cope well. They also note that 7 out of 9 noncompliant patients were geographically distant from the hospital at the time of their noncompliance. Frierson and Lippman discuss 70 patients who were evaluated for transplantation, 13 of whom were rejected on the basis of psychiatric criteria (2). History of noncompliance or poor compliance was the most frequent reason for rejection. The diagnoses among the 13 patients who were rejected included substance abuse, clinical depression, antisocial personality disorder, borderline intellectual functioning, schizophrenia, somatization disorder, and delusional disorder. One patient with antisocial personality was accepted for transplantation, but had difficulty adjusting postoperatively, had conflicts with staff, and was noncompliant. Out of 5 patients with a preoperative diagnosis of substance abuse, 2 required postoperative treatment for continued substance abuse and all of them reported craving (for alcohol). Two patients with a preoperative diagnosis of affective disorder became suicidal after the transplantation and required psychiatric hospitalization and treatment with antidepressants. The authors feel full commitment to the preoperative medical regimen is a good indicator of future compliance. They recommend every cardiac transplant team should receive psychiatric input, the waiting period should be considered an extension of the evaluation period, and definite psychiatric disqualifiers should be established. They also state recognition of the psychological stress on the decision makers is essential and suggest regularly scheduled debriefing sessions for members of the transplant team.

Allender, et al., identify the patient's ability to hold up under the stress of cardiac transplantation as a major concern of the preoperative evaluation and hold that how a patient will react to stress can often be predicted from his/her reactions to previous life stressors and from the results of preoperative psychological testing (10). A high degree of somatic anxiety reflected on psychological test profiles may indicate an impaired ability to cope with the various challenges of cardiac transplantation (10).

Issues to consider during the evaluation period include patient's anxiety about making a decision and the acceptance of the transplant team, patient ambivalence, and informed consent $(5,13)$. Since unexpressed emotions can produce counterproductive behaviors, patients need to be encouraged to express their feelings, concerns, and questions (5). Some patients may not actually want to have transplantation but may be pushed by external factors such as family pressure. This ambivalence can manifest itself in indirect ways such as noncompliance, presenting obstacles to further assessment and treatment $(1,13)$. Before giving informed consent for surgery, patients need to become familiar with the transplant protocol and understand what lies ahead for them including the financial and social implications of cardiac transplantation $(3,13,14)$. Good patient education is very important at this stage.

Patients who are not accepted as candidates for cardiac transplantation may react to this in a variety of ways. Common reactions include refusal to leave the 
hospital, angry outbursts directed at staff, threats of litigation, decision to seek transplantation elsewhere, and expressions of relief (7). At the University of Lousville program all patients rejected for psychiatric reasons were offered continued psychiatric care, but only a few accepted (7).

\section{Waiting Period}

This period begins when the patient receives the news that he or she has been accepted as a transplant candidate and will be placed on the waiting list $(5,10,13)$. During the initial phases of this period, the patient is completing a busy evaluation period culminating in acceptance to the transplant program. Many patients begin to think that there may be some hope for a solution to their crippling and potentially lethal disease and this is often accompanied by euphoria (5). However, since the number of available hearts is far short of the number of qualified candidates, this period can be long and fraught with problems both for the patients and transplant staff (3). Levenson and Olbrisch address the problems that may arise in this period (3). They note that the waiting period is often marked by significantly decreased contact with the transplant staff. Concurrently, patients' concerns and anxiety regarding transplant surgery and the possibility of death begin to peak and as the wait stretches from days to weeks patients may start feeling more and more isolated, abandoned, and even forgotten (3). The transplant team physicians often feel they have nothing to offer and may be reluctant to see the waiting patients until a donor organ is available (3). Prolonged waiting periods may result in patients ruminating about circumstances that may increase the chances of a heart being available such as bad weather conditions ("donor weather") which is often followed by guilty feelings (5). Levenson and Olbrisch cite "gallows humor" as one of the first manifestations of decreased coping ability by patients and their families. An example of this would be patients fantasizing about standing on the roof of the hospital with a rifle or asking the hospital staff whether they have had any opportunities to run down pedestrians on their way to work (3). The strain of waiting, along with patient and family misconceptions, can erupt in anger and frustration which, unfortunately, is often directed at staff members who have been most supportive and least avoidant because they are the most available ones. This may complete a vicious circle creating more reasons for staff to spend less time with patients and their families (3).

To alleviate the potential problems during the waiting period referred to above, Levenson and Olbrisch suggest careful preoperative patient education including organ procurement and assignment factors (3). They state staff should avoid any appearance of trying to elude the waiting patients and maintaining regular supportive contact while being careful not to inadvertantly reinforce patient misconceptions such as by seeming to encourage "VIP" treatment for selected patients. They also recommend group meetings for waiting families led by a member of the transplant team, and programs for staff explaining the waiting stresses and the dynamics of staff, patient, and family interactions.

The perioperative period extends from the time a suitable heart is found and 
surgery preparations are initiated until the patient is transferred out of the ICU postoperatively (5). Availability of a donor heart often brings with it relief and a sense of serene calm $(5,1)$. Immediately after the operation euphoria and a feeling of "being reborn" are common (5). It is unusual to have major psychological complications at this stage $(5,10,13)$.

\section{POST-TRANSPLANT PERIOD}

\section{In-hospital Convalescence}

This stage begins when the patient is transferred to the regular medical floor from the ICU and continues until the patient is discharged from the hospital (5). After the initial euphoria, the tedium of posttransplantation routines and regimens set in. Patients often start this stage with a feeling of specialness and victory over death until the time of the first endocardial biopsy which introduces the possibility of graft rejection and marks the end of the postsurgical "honeymoon" period $(5,13)$. Watts, et al., recommend premedication with hypnotics the night prior to and anxiolytics the day of the first endocardial biopsy (6). In the event of a complication such as infection or transplant rejection, the patient may become despondent and feel that one problem has been traded for another (13). Anxiety can lead to problems such as frustration and low pain tolerance (10). Patients may experience thoughts of dying and feelings of helplessness as well as anger and frustration at themselves because they may see themselves as not perfect enough in following medical direction to avoid rejection (13). Feelings of depression often begin to resolve as the graft rejection episode resolves (10). If the patient can maintain sustained improvement, it leads to trust in the ability to survive crises with the new heart (5). Around this time, patients may fantasize over whose heart they may have. Initially, the new heart is regarded as a foreign object of which they are always conscious but awareness dissipates over time and only returns when patients are reminded of it (5). Castelnuovo-Tedesco addresses the problems in the incorporation of a transplanted organ and refers to Margaret Mahler's symbiotic phase when the infant initially cannot distinguish his body parts from that of his mother, but subsequently learns to differentiate himself from the mother's body (15). Upon transplantation, suddenly parts from another human being are introduced into the patient's body. This may undo a lifelong process of differentiation and the author sees this as having a tremendous impact on the ego which can be very disruptive and even give rise to psychosis (15). During the stage of inhospital convalescence, patients are usually integrated into a network of previous transplant patients and waiting candidates. Having something to offer newcomers into the transplant program tends to increase self esteem (5).

A study from the University of Western Ontario explores the occurence of graft and donor denial during the period of inhospital convalescence (16). Postoperative follow up of 20 cardiac transplant patients revealed 18 out of 20 had at least some denial regarding graft and/or donor. Seven patients had graft denial and made 
statements such as "I have no thoughts about the new heart" or "I try to forget about the new heart." Five patients had donor denial, for example: "I have not asked where the heart came from" or "I know the donor was 34 but I don't want to know anymore, it may bother me." 6 patients had both graft and donor denial. Feelings expressed by the group of patients involved in this study included euphoria, gratitude, ambivalence regarding the donor, guilt (over being alive and the donor being dead), anxiety, and a feeling of a change in body image. Mai suggests denial may be a means of coping with or postponing these feelings until they can be better accommodated and that staff should encourage this process of denial postoperatively (16). The findings above are consistent with the findings of Mai et al., who found that $90 \%$ of the transplant survivors expressed overt disinterest and denial regarding the graft and donor (8).

When patients and their families find out information about the donor, they may start to fear that the patient is assuming the characteristics of that person (7). For example, the wife of one patient complained that her husband became more feminine after receiving a woman's heart (7). This may even occur with some hospital staff; a nurse who was working with a noncompliant patient who had received a physician's heart stated in all earnestness "Someone with his medical background should know better than to refuse medication" (7). On the other hand, the donor family may feel part of their family member is alive in the transplant recipient's body and may maintain an intense emotional involvement with the transplant recipient (4). One family seemed to experience a delayed grief reaction when the transplant patient died. The death of the heart seemed to finalize the death of the donor for this family (4).

If everything goes according to plan, usually somewhere between the 30th and the 90 th postoperative day the patient is discharged from the hospital (16). Although patients may state that they are ready to go home, anxiety about leaving the dependency of 24 hour care in the hospital is often present $(5,10)$. Reestablishing closer relationships with family and friends becomes important and out-of-hospital support systems are considered critical for successful recovery (5). Occasionally, patients' families also express anxiety at this stage and may need support and reassurance from the transplant staff (10).

\section{Postdischarge Adaptation}

After leaving the hospital, patients face the adaptive task of readjusting to the outside world. They often remain engaged in a supportive network of "transplant graduates" and make themselves available to the newly hospitalized transplant candidates (5). Slowly, they will begin to see themselves less and less as transplant patients and most will report feeling as though the new heart had always been theirs (5). The first anniversary of the transplant operation is regarded as a major milestone; prior to this full readjustment usually does not occur (5). During this stage, it is critical for the patient to comply carefully with the prescribed medical regimen; agents such as immunosuppressants and steroids must be taken even if they produce undiserable side effects such as changes in appearance (7). Cooper et al., 
emphasize that playing on the patient's fears in the hopes of increasing compliance is frequently ineffective and recommend staff recognition, clarification, and definition of the patient's own beliefs about his illness, the patient's ideas about the treatment and its side effects, and changes in life style to improve compliance rates (12). Frequent clarifications and reassurance by staff members may be necessary (12).

In the posttransplantation period, about one third of the transplant families run into significant family and marital problems and these problems typically tend to be exacerbations of previous difficulties that have simply become more acute due to the stress of the transplant process (10). Problems may arise, for example, if the patient insists on being treated as an invalid or the family, accustomed to an incapacitated patient, may not be ready for the patient to assume a more active role in the family or reclaim a previous leadership position (10). Patients may also experience sexual dysfunction which is often due to impotence in men treated for cyclosporine-related hypertension (14). Professional help in the form of marital and family counseling may be required if the family and marital problems persist (10).

A number of studies have looked at the incidence and prevalence of psychiatric disorders in transplant patients both pre- and postoperatively. A study from Columbia University looked at 73 transplant patients comprising two groups (14). One group of 30 patients was evaluated prospectively, undergoing both preoperative evaluation and postoperative follow-up from 2 weeks to 12 months after surgery. The other group was comprised of 43 patients who had already had the transplant surgery performed. They were evaluated in the follow-up period only, which ranged from 6 months to 6 years postoperatively. When the postoperative results for both groups were combined, overall $51 \%$ of all patients were diagnosed as having affective illness which also included steroid related syndromes. Major depression occurred in $11 \%$ of all patients postoperatively. Significant anxiety symptoms occurred in $26 \%$ of patients with both groups combined but only $17 \%$ of the follow-up group and $22 \%$ of the prospective group qualified for a clinical anxiety disorder. Other problems included family/marital problems, sexual dysfunction, noncompliance, body image problems, and substance abuse. Shapiro and Kornfeld note in their discussion that a history of major depressive episode(s) and even clinically significant depression at the time of the preoperative evaluation did not predict a major adverse outcome psychiatrically or otherwise (14).

Freeman et al., looked at three components and their relationship to outcome: pretransplant evaluation, postoperative complication assessment, and longitudinal measurements of anxiety, depression, psychosocial adjustment, and cognitive functioning (9). These longitudinal determinations were made within the first and second 6 month periods following the transplant operation and annually thereafter. The tests utilized included MMSE, Spielberger state anxiety inventory, Zung self depression scale, and psychosocial adjustment inventory scale. Out of a total of 70 patients, 26 were given axis I and 8 were given axis II diagnoses. Reservations were expressed on 19 patients preoperatively. This group included history of clinical depression, sociopathy, substance abuse, and passive-aggressive, dependent, and histrionic personality traits. Of those 19 patients, 7 died perioperatively, 7 developed significant 
psychiatric morbidity requiring assessment and intervention, and 5 did not have any notable problems postoperatively. The article does not discuss the postoperative course of patients for whom no reservations were expressed. Psychiatric problems requiring consultation and intervention occurred in 24 out of 70 patients. The most frequent postoperative psychiatric complication was organic mental disorder which was diagnosed in 17 patients; of these, 10 were judged to be steroid related syndromes. The second most frequent psychiatric complication was clinical depression (8 patients). The longitudinal assessments showed overall improvement in the mean test scores over time. The authors note that postoperative mood disturbances were more frequent in individuals with a prior history of depression, family history of affective disorders, or personality disorders which appears to contradict the findings of Shapiro and Kornfeld which was discussed earlier (14).

Freeman et al., list 8 case vignettes regarding patients who underwent cardiac transplantation and note that those patients who did not survive postoperatively had a high incidence of both pre and postoperative anxiety and depression (1). The authors postulate that there may be some relationship between psychiatric factors and postoperative outcome possibly mediated by immune factors and state further studies are needed to explore this relationship.

Watts et al., divide the postoperative psychiatric complications into three major categories: anxiety disorders, major depressive episodes, and organic brain syndromes (6). They emphasize that the list of differential diagnoses in the assessment of mental status changes in the transplanted patients often includes medical complications (anxiety secondary to arrhythmias, depression secondary to hypothyroidism, depression and/or organic brain syndrome secondary to opportunistic infections, etc.) and that symptoms may be secondary to medical complications rather than a primary psychiatric problem. For example, Surman notes infection is a common cause of post-transplant depression which is especially prevalent in patients with CMV infections (17). Therefore, when a psychiatrist is called upon to evaluate mental status changes in a transplant recipient, in addition to evaluating the patient's premorbid personality, preoperative emotional adjustment, postoperative anxiety regarding rejection, and postoperative environmental factors (such as reverse isolation), he/she also needs to consider underlying organic factors including corticosteroid treatment which confers a dose-related risk typically in doses greater than $40 \mathrm{mg}$ of prednisone equivalent per day (6). Ling et al., note that although steroid dosages may correlate with risk of developing steroid-induced mental disturbances, time of onset, duration, severity, and types of such mental disturbances seem to be independent of steroid dosages or duration of treatment (18). They cite affective symptoms such as hypomania, agitation, confusion, euphoria, mood lability, depression, and psychotic reactions such as delusions and hallucinations as common manifestations of steroid-induced mental status changes and note these changes are usually reversible through dose reduction or discontinuation of steroids (18). Surman discusses some of the possible adverse effects of cyclosporine which has become the mainstay of antirejection therapy (17). In addition to causing potential hypertension, cyclosporine can have adverse effects on the CNS which can include seizures, 
encephalopathy, and organic brain syndromes. A few patients have experienced cortical blindness, quadriplegia, confusion and coma which were reversed by dose reduction or discontinuation of cyclosporine therapy (17).

With respect to treating postoperative psychiatric complications of nonorganic origin, Surman maintains the consultation psychiatrist should provide supportive interventions during periods of family and medical crises (17). He notes that adjustment-related depression should initially be treated supportively and when symptoms persist antidepressant therapy may be indicated with the dosages adjusted according to the patients' liver and kidney function. Postoperative organic brain syndromes usually can be treated with medical intervention and by adjusting the dose of immunosuppressants (17). Surman also notes the presence of a relative or close friend at the patient's bedside may eliminate any need for antipsychotic medication.

\section{CARDIAC VERSUS OTHER ORGAN TRANSPLANTATIONS}

One of the unique aspects of cardiac transplantation compared to other organ transplantations stems from the fact that the heart carries a special symbolic significance in our society (4). The heart is often associated with emotions as well as strength and intensity of feelings (16). As a result of this, I believe identity disturbances referred to above are more common in cardiac transplant recipients and the issues of incorporation addressed by Castelnuovo-Tedesco (15) may become more problematic. Surman notes that while end-stage renal disease patients may opt for dialysis instead of transplantation, those with end-stage cardiac and liver disease can only rely on temporary medical supports (17) and this, in my opinion, may exacerbate tensions and pose greater risk of maladaptive behaviors for heart and liver transplant candidates during the waiting period. Also, unlike transplants involving heart and liver, some kidney transplant recipients may receive their kidney from a living donor which introduces its own set of circumstances and psychological sequelae (19). To my knowledge, there have been no articles to date that have specifically compared and discussed the unique aspects of different organ transplantations.

\section{CONCLUSION}

The articles reviewed in this series are unanimous in endorsing the involvement of psychiatry in the cardiac transplant programs both pre and postoperatively. Most consider it essential and some highly recommend that psychiatric assessment be a regular part of the transplant process. As Allender et al., indicate (5), few patients have trouble with all the stages of transplantation but it appears each patient reacts to at least one stage more than the other patients in general. Psychiatric assessment in the evaluation stage can be valuable in determining which patients are appropriate cardiac transplant candidates $(14,4,2)$. A substantial proportion of transplant patients develop psychiatric complications as a direct result of medications needed to prevent graft rejection or in response to situational factors potentially related to the 
transplant (13). An advantage of having psychiatry routinely involved is early recognition and treatment of psychiatric problems.

\section{ACKNOWLEDGEMENTS}

The author would like to express his thanks and appreciation to Stuart Youngner, MD and David Agle, MD for their support and critical review of this paper.

\section{REFERENCES}

1. Freeman AM, Watts D, Karp R: Evaluation of cardiac transplant candidates: Preliminary observations. Psychosomatics 25:197-207, 1984

2. Mai FM: Liaison psychiatry in the heart transplant unit. Psychosomatics 28:44-46, 1987

3. Levenson JS, Olbrisch ME: Shortage of donor organs and long waits. Psychosomatics 28:399-403, 1987

4. Lunde DT: Psychiatric complications of heart transplants. Am J Psychiatry 126:369-373, 1969

5. Kuhn WF, Davis MH, Lippman SB: Emotional adjustment to cardiac transplantation. General Hospital Psychiatry 10:108-113, 1988

6. Watts D, Freeman AM, McGiffin DG, Kirklin JK, McVay R, Karp RB: Psychiatric aspects of cardiac transplantation. Heart Transplantation 3:243-247, 1984

7. Frierson RL, Lippman SB: Heart transplant candidates rejected on psychiatric indications. Psychosomatics 28:347-355, 1987

8. Mai FM, McKenzie NF, Kostuk WJ: Psychiatric aspects of cardiac transplantation: preoperative evaluation and postoperative sequelae. Br Med J 292:31 I-313, 1986

9. Freeman AM, Folks DG, Sokol RS, Fahs JJ: Cardiac transplantation: Clinical correlates of psychiatric outcome. Psychosomatics 29:47-54, 1988

10. Allender J, Shisslak C, Kaszniak A, Copeland J: Stages of psychological adjustment associated with heart transplantation. Heart Transplantation 2:228-231, 1983

11. Merrikin KJ, Overcast TD: Patient selection for heart transplantation: When is a discriminating choice discrimination. J of Health Politics, Policy and Law 10:7-32, 1985

12. Cooper DKC, Lanza RP, Barnard CN: Noncompliance in heart transplant recipients: The Cape Town experience. Heart Transplantion 3:248-253, 1984

13. Christopherson LK: Cardiac transplantation: a psychological perspective. Circulation 75:57-62, 1987

14. Shapiro PA, Kornfeld DS: Psychiatric outcome of heart transplantation. Gen Hosp Psychiatry 11:352-357, 1989

15. Castelnuovo-Tedesco P: Ego vicissitudes in response to replacement or loss of body parts. Psychoanal Q 47:381-397, 1978

16. Mai FM: Graft and donor denial in heart transplant recipients. Am J of Psychiatry 143:1159-1161, 1986

17. Surman OS: Psychiatric aspects of organ transplantation. Am J Psychiatry 146:972-982, 1989

18. Ling M, Perry P, Tsuang M: Side effects of corticosteroid therapy. Arch Gen Psychiatry $38: 471-477,1981$

19. Simmons RG, Klein SD: Family noncommunication: The search for kidney donors. Am J Psychiatry 129:687-692, 1972 